\title{
Functional polymorphisms within the TNFRSF11B (osteoprotegerin) gene increase the risk for low bone mineral density
}

\author{
Christopher Vidal $^{1,2}$, Robert Formosa ${ }^{1,2}$ and Angela Xuereb-Anastasi ${ }^{1,2}$ \\ ${ }^{1}$ DNA Laboratory, Department of Applied Biomedical Science, Faculty of Health Sciences and ${ }^{2}$ Department of Pathology, Faculty of Medicine and Surgery, University of Malta, \\ Msida, Malta \\ (Correspondence should be addressed to A Xuereb-Anastasi at DNA Laboratory, Department of Applied Biomedical Science, Faculty of Health Sciences and Department of Pathology, \\ Faculty of Medicine and Surgery, University of Malta; Email: angela.a.xuereb@um.edu.mt)
}

\begin{abstract}
Polymorphisms within the TNFRSF11B gene have been studied and associated with osteoporosis and fracture risk. Osteoprotegerin (OPG), the product of this gene, is a key negative regulator of osteoclastogenesis and is secreted by osteoblasts/stromal cells. A previous study in Maltese postmenopausal women showed positive association of low bone mineral density (BMD) with a polymorphism found within the promoter region of this gene (C950T). In this study, direct DNA sequencing revealed 12 variants with polymorphisms C950T, G1181C and rs4876869 observed to be in strong linkage disequilibrium. The constructed haplotype T-G-T was found to increase the risk for a low BMD, while C-G-T and $\mathrm{C}-\mathrm{C}-\mathrm{C}$ have a protective role; thus, we investigated the functional role of both C950T and rs4876869 in vitro. The promoter region, including the C950T alleles, was amplified by PCR, cloned into pGL3 enhancer vector and transfected into HeLa, COS-7 and RAW264.7 cell lines. After incubation, luciferase activity was measured. The T/C (rs4876869) change was tested for its possible effect on pre-mRNA splicing, using an exon-trapping vector. A statistical significant difference in gene expression was observed between the alleles for T950C, with the T allele showing a lower luciferase expression in all cell lines $(P<0 \cdot 01)$. For rs4876869, exon skipping was observed for the $C$ allele, while only one transcript harbouring the whole exon was observed for the T allele. Our findings suggest that the T-G-T haplotype might be increasing the risk for osteoporosis due to lower quantities of the full OPG transcript being expressed resulting in a higher bone resorption.
\end{abstract}

Journal of Molecular Endocrinology (2011) 47, 327-333

\section{Introduction}

With an increase in the ageing population worldwide, late onset chronic disorders such as osteoporosis, heart disease and diabetes become a greater economic burden on society. These disorders are said to be complex because they are caused by interactions of various environmental and genetic factors. Osteoporosis is a chronic complex disorder characterised by loss of bone mineral density (BMD), deterioration of bone micro-architecture and increased risk for fractures. For more than a decade, a large number of genetic studies have been performed using different approaches to try to identify genetic variants responsible for susceptibility to disease and to understand better bone physiology at the molecular level. Unfortunately, most of these studies led to inconclusive and conflicting results, with the exceptions of the collagen type I (COL1A1) and lipoprotein receptor-related protein $(L R P)-5$ genes that have been repeatedly associated with BMD and fractures (Xu et al. 2009). A large number of polymorphisms, found within candidate genes involved in bone physiology, were studied for association using case-control studies; however, functional studies of the same polymorphisms are lacking.

One of the most studied genes is TNFRSF11B, found on chromosome 8q23-24 and encoding osteoprotegerin (OPG). OPG secreted by osteoblasts/stromal cells in the bone micro-environment is a negative regulator of osteoclastogenesis, which binds to RANKL, thus preventing activation of osteoclast precursors through RANK (Hofbauer et al. 2000, Boyle et al. 2003). Several polymorphisms were studied in the promoter region and elsewhere within this gene in different population for association with various osteoporosis-related phenotypes as well as with other human disorders (Hofbauer \& Schoppet 2002, García-Unzueta et al. 2008, Park et al. 2008, Jurado et al. 2010). The locus harbouring the TNFRSF11B gene was also associated with low BMD and fracture risk by genome-wide association studies (GWAS; Richards et al. 2008, Styrkarsdottir et al. 2008). Functional studies investigating allele-specific expression of TNFRSF11B transcript confirmed the association data from GWAS (Richards et al. 2008). 
In a previous study, we reported a significantly higher frequency of haplotype A-T-G within the TNFRSF11B gene promoter in postmenopausal women with low BMD (Vidal et al. 2006). Genotype distribution was significantly different for the C950T polymorphism, with TT genotype found in $83 \%$ of postmenopausal women with low BMD and an odds ratio of $2 \cdot 11$ for the T risk allele. Individual polymorphisms might increase the risk for disease either because they are functional themselves or because they are in linkage disequilibrium (LD) with a functional polymorphism within the same or a nearby gene. However, the functionality of these polymorphisms was not assessed. Thus, the aim of this study was to identify and investigate the functional role of polymorphisms within the TNFRSF11B gene in a group of postmenopausal women with normal and low BMD.

\section{Materials and methods}

\section{Subjects}

One hundred and forty-seven postmenopausal women were recruited from new subjects referred by medical practitioners to the Bone Density Unit at the Department of Obstetrics and Gynaecology, St. Luke's Hospital, Malta, for an osteoporosis risk evaluation. All subjects studied were healthy Caucasian women with the age of $40-75$ years, with a mean ( \pm s.D.) age of $55 \cdot 7 \pm 6 \cdot 9$ years. Exclusion criteria were the same as those described in a previous study on the same but slightly extended group of postmenopausal women (Vidal et al. 2006). Menopause was defined as amenorrhoea of at least 6 months duration. Blood for DNA analysis was collected by venipuncture in a tube containing $\mathrm{K}_{2}$-EDTA as an anticoagulant. BMD was measured at the lumbar spine (LS; L2-L4) and femoral neck (FN) using a Norland 486 dual-energy X-ray absorptiometer (Norland, Medical Systems, Inc., New York, NY, USA). Our sample was divided into two main groups: one of normal individuals $(40 \cdot 1 \%)$ having LS $t$-score $>-1.0$ and a group of individuals that were osteopenic and/or osteoporotic at the LS (59.9\%). At the FN, $56.5 \%$ had normal BMD and $43.5 \%$ had low FN BMD.

\section{TNFRSF11B gene sequencing}

All exons, including intron-exon boundaries, and up to $1 \mathrm{~kb}$ upstream from the transcriptional start sites of the TNFRSF11B (ENST00000297350) gene, were sequenced. Sequencing reactions were performed using BigDye Terminators v3.1 and analysed on an ABI 3130 genetic analyser (Applied Biosystems, Foster City, CA, USA). Sequencing was initially performed in
20 postmenopausal women. Following initial sequencing, a T/C change in intron 2 (rs4876869) that was in $\mathrm{LD}$ with the C950T variant was determined in 104 postmenopausal women by direct DNA sequencing. Nomenclature used refers to actual nucleotide changes in the respective polymorphisms.

\section{TNFRSF11B SNP functional assays}

\section{Preparation of constructs}

To test whether the C950T single nucleotide polymorphism (SNP) affects gene expression, a luciferase reporter assay was used. A construct, harbouring either of the C950T alleles, was designed and amplified by PCR using oligonucleotide primers harbouring consensus sequences for restriction enzymes KpnI and NheI and a non-specific sequence at the $5^{\prime}$-end. The construct was labelled as pGL3E-C950T and amplifies up to nucleotide 341 upstream from the initial codon. PCR was performed to amplify both normal and mutated alleles for each construct using a DNA sample from a heterozygous individual for this variant. PCR products were checked using $1 \%$ agarose gel electrophoresis, cleaned and digested using restriction enzymes KpnI and NheI (New England Biolabs, Beverly, MA, USA) in a $50 \mu \mathrm{l}$ reaction. These fragments together with digested pGL3 enhancer vector (Promega Corporation) were cleaned from agarose gel and ligated overnight at $4{ }^{\circ} \mathrm{C}$ using $\mathrm{T}_{4}$ DNA ligase (Promega Corporation), following manufacturer's instructions. Transformation was performed in chemically competent DH5 $\alpha$ (Invitrogen Ltd), using a heat-shock protocol. Extracted plasmids were checked by restriction digestion for the presence of insert and sequenced using the BigDye Terminator kit v3.01 (Applied Biosystems) to verify that the insert with the correct sequence was cloned and to select normal and mutated alleles for each construct. Highly purified plasmid DNA for transfections was prepared using PureYield Plasmid Midiprep System (Promega Corporation). The plasmid DNA concentration was measured by spectrophotometry and adjusted to $200 \mu \mathrm{g} / \mathrm{ml}$.

To test whether the $\mathrm{T} / \mathrm{C}$ change in intron 2 (rs4876869) affects pre-mRNA splicing, the exon trapping vector pSPL3 (generously donated by Prof. Junko Oshima, Department of Pathology, University of Washington, Washington, USA) was used. Mutated and wild-type alleles were amplified by PCR using DNA from a heterozygous individual as template using oligonucleotide primers harbouring consensus sequences for restriction enzymes XhoI and PstI. An amplicon of 489 bp was amplified consisting of exon 3 and part of adjacent introns, which was cloned into pSPL3 vector, transfected into HeLa, COS-7 and RAW264.7 cell lines and analysed as described before (Vidal et al. 2009). 
The same cell lines were used for luciferase reporter assays. All experiments were done in triplicate.

\section{Luciferase reporter assay}

The assay was performed using the Dual-Luciferase Reporter System (Promega Corporation) following manufacturer's instructions. The results were normalised for transfection efficiency using the firefly to renilla luciferase ratio. The promoter activity for each construct was expressed as fold increase over baseline of empty vector. Statistical comparisons between reporter gene expression of different constructs and between mutated and normal alleles were performed using a $t$-test, and the Bonferroni correction was applied for multiple comparisons.

\section{Statistical analysis}

The EH Software by Terwillinger \& Ott (1994) (ftp:// linkage.rockefeller.edu/software/utilities) was used to construct haplotypes of polymorphisms within the same gene to test for association between alleles (LD) and for association of haplotypes with low BMD by comparing the normal with the low BMD.

All statistical tests were performed using the Statistical Package for Social Sciences (SPSS) for Windows student version 9.0 (SPSS, Chicago, IL, USA). All tests were considered two tailed at a level of significance of 0.05 where the null hypothesis (no difference between means) was rejected at $P<0 \cdot 05$.

\section{Results}

\section{TNFRSF11B gene sequencing}

Direct DNA sequencing of the TNFRSF11B gene in 20 postmenopausal women revealed 12 sequence variants: A163G (rs3102725), T149C (rs3134071), G209A and
T245G all within $1 \mathrm{~kb}$ from the transcriptional start site, the C950T (rs2073617) and a C/T transition in the untranslated region of exon 1 . Other variations include G1181C (rs2073618) in exon 1 (signal peptide), C445T (rs1564858), a T/C transition in intron 2 (rs4876869), $\operatorname{del}(\mathrm{CT})$ (rs10554146), A6833G (rs2228568) and A6890C (rs7844539). Strong LD was observed between A163G, G209A and T245G $\left(\chi^{2}=40 \cdot 0 ; P<0 \cdot 0001\right)$ and of C445T with del(CT) $\left(\chi^{2}=40 \cdot 0 ; P<0 \cdot 0001\right)$, A6833G and A6890C $\left(\chi^{2}=29 \cdot 2 ; P<0 \cdot 0001\right)$. Strong LD was also observed between C950T, G1181C and rs4876869 $(P<0 \cdot 0001)$. Since rs4876869 was in LD with C950T $\left(\chi^{2}=13 \cdot 2 ; P=0 \cdot 01\right)$, previously associated with low $\mathrm{BMD}$, genotyping was performed in 104 postmenopausal Maltese women.

\section{Rs4876869 and BMD}

Genotype frequencies observed for rs4876869 T/C transition in Maltese postmenopausal women were TT: $26.9 \%$, TC: $53.8 \%$, CC: $19 \cdot 3 \%$ and were in Hardy-Weinberg equilibrium $\left(\chi^{2}=0 \cdot 67 ; P=0 \cdot 412\right.$; $\mathrm{df}=1$ ). As shown in Table $1, \mathrm{rs} 4876869$ did not have any effect on lumbar or FN BMD $(P>0.05)$ even after adjusting for age and body mass index (BMI). The distribution of genotype frequencies between normal postmenopausal women and those having a low BMD was not statistically significant $\left(\chi^{2}=0 \cdot 34\right.$; $P=0 \cdot 843 ; \mathrm{df}=2)$.

When constructed haplotypes for C950T, G1181C and SNP rs $4876869,42 \cdot 4 \%$ of postmenopausal women carried haplotype C-C-C, 38.5\% T-G-T and 7\% haplotype C-G-T. Comparing haplotype frequencies between women with normal BMD and those having a low $\mathrm{BMD}$, statistical significance was reached $\left(\chi^{2}=18 \cdot 42\right.$; $P=0 \cdot 010 ; \mathrm{df}=7)$. Haplotype T-G-T increases the risk for low BMD (35.9\% of normal vs $41.0 \%$ of women with low BMD) while haplotypes C-G-T and C-C-C have a protective role.

Table 1 General characteristics, lumbar and femoral bone mineral density (BMD) in Maltese postmenopausal women according to rs4876869 polymorphism. $P$ values obtained by ANOVA or independent sample $t$-test

General characteristics and genotypes

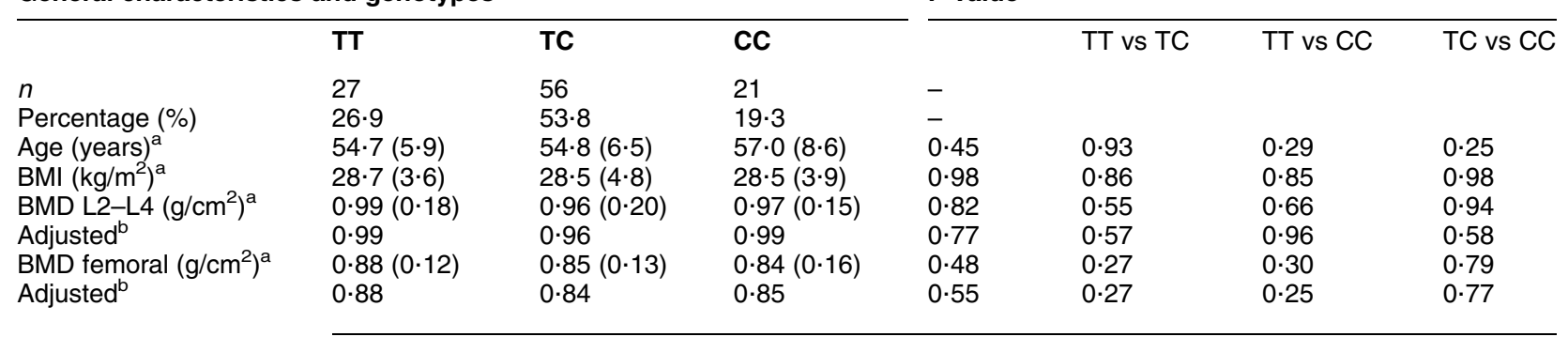

${ }^{a}$ Values are means \pm S.D. in parentheses.

${ }^{b}$ Values are adjusted for age, BMI and years since menopause; 95\% confidence intervals given by univariate analysis (not shown). 


\section{C950T SNP reporter assay}

Luciferase reporter assays show that gene expression was affected by the C950T polymorphism. As shown in Fig. 1, gene expression was significantly higher in the presence of the $\mathrm{C}$ allele in all cell lines tested. More than a twofold difference was observed between the two alleles in RAW264.7 cell $(P<0 \cdot 001)$. Difference between the alleles remained significant even after Bonferroni correction for multiple comparisons.

\section{Pre-mRNA analysis of rs4876869}

Using an exon-trapping vector (pSPL3), we assessed whether the $\mathrm{T} / \mathrm{C}$ change in intron 2 (rs4876869) affects pre-mRNA processing. As shown in Fig. 2, two transcripts (453 bp and $295 \mathrm{bp}$ ) were amplified by RT-PCR in the presence of the $\mathrm{C}$ allele, while only one transcript (453 bp) consisting of vector exons and the entire TNFRSF11B exon 3 was amplified in the presence of the $\mathrm{T}$ allele. Results were consistent for all cell lines used. Sequencing of all transcripts confirmed the presence of the entire exon 3 in the biggest transcript (453 bp) and the absence of almost the entire exon from the $295 \mathrm{bp}$ fragment amplified for the $\mathrm{C}$ allele (Fig. 2), confirming that the $\mathrm{C}$ allele affects pre-mRNA splicing in this model.

\section{Discussion}

OPG, encoded by the TNFRSF11B gene, is a key negative regulator of osteoclastogenesis. Although a number of polymorphisms were studied for association with low BMD, functional analysis of these SNPs is lacking. Gene expression was reported to be affected by a haplotype within the TNFSF11 (RANKL) gene showing association with LS BMD (Mencej et al. 2008).
OPG, a negative regulator of osteoclastogenesis, binds to RANKL preventing it from activating RANK. In this study, we focused on three SNPs within the TNFRSF11B gene that were in strong LD with each other, since genotype frequencies for one of these SNPs (C950T) were associated with an increased risk for low BMD (Vidal et al. 2006). Polymorphisms that show positive association with a disease can either be functional themselves or else are in LD with another nearby variant. To evaluate the roles of SNPs C950T and rs4876869, functional assays were performed to assess gene expression and pre-mRNA splicing. Reporter assays showed that the T allele of SNP C950T negatively affects gene expression. Our observations were supported by a higher frequency of the TT genotype in postmenopausal women with low BMD suggesting that C950T affects bone mass by decreasing OPG expression by osteoblasts/stromal cells in the bone microenvironment. These results agree with those reported by Hagedorn et al. (2009), where it was shown that transcriptional factor early growth response (Egr)-1 binds exclusively to the $\mathrm{T}$ allele, preventing specific protein (Sp)-1 binding and thus acting as a repressor. However, SNP C950T alleles were not always correlated with serum OPG levels and bone mass, since systemic OPG could be independently influenced by a number of other factors (Ueland et al. 2007). This suggests that the effect on bone might only be related to the levels of expressed OPG secreted by osteoblasts/stromal cells within the bone micro-environment. As previously reported (Vidal et al. 2006), although TT homozygotes had the lowest BMD, no significant association was found between C950T and BMD as a continuous (quantitative) variable, but this trend was similar to that of other larger studies (Brandstrom et al. 2004, Ueland et al. 2007). The reason for this might be due to the low sample power due to a small sample size, since one would require a much bigger sample to detect
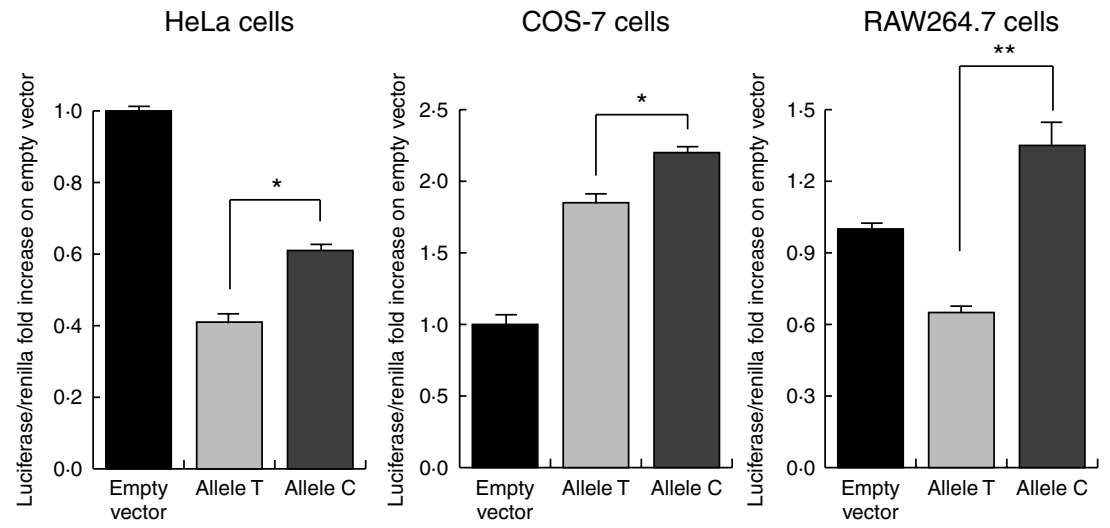

Figure 1 Effect of C950T SNP on luciferase gene expression in all three cell lines tested. A statistically significant higher gene expression was observed for the $C$ allele when compared with the T allele ( $t$-test, $\left.{ }^{\star} P<0 \cdot 01 ;{ }^{\star \star} P<0 \cdot 001\right)$. 
A
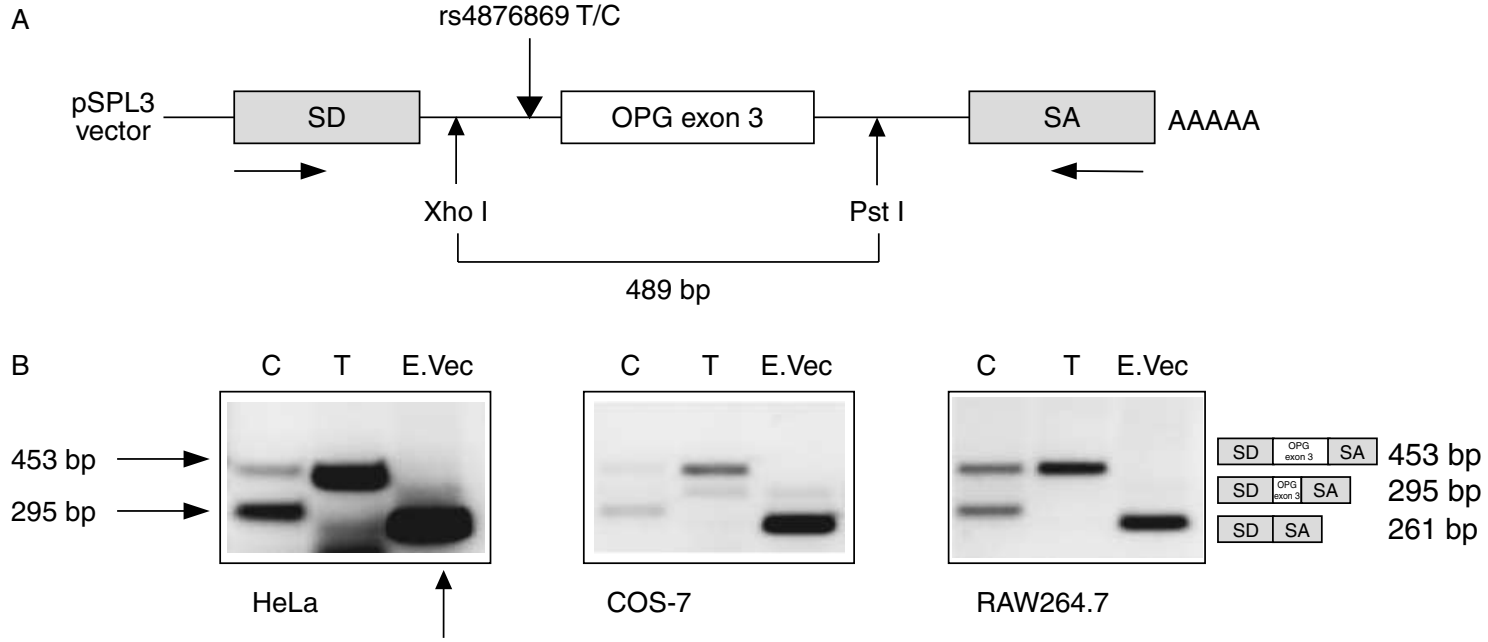

$261 \mathrm{bp}$

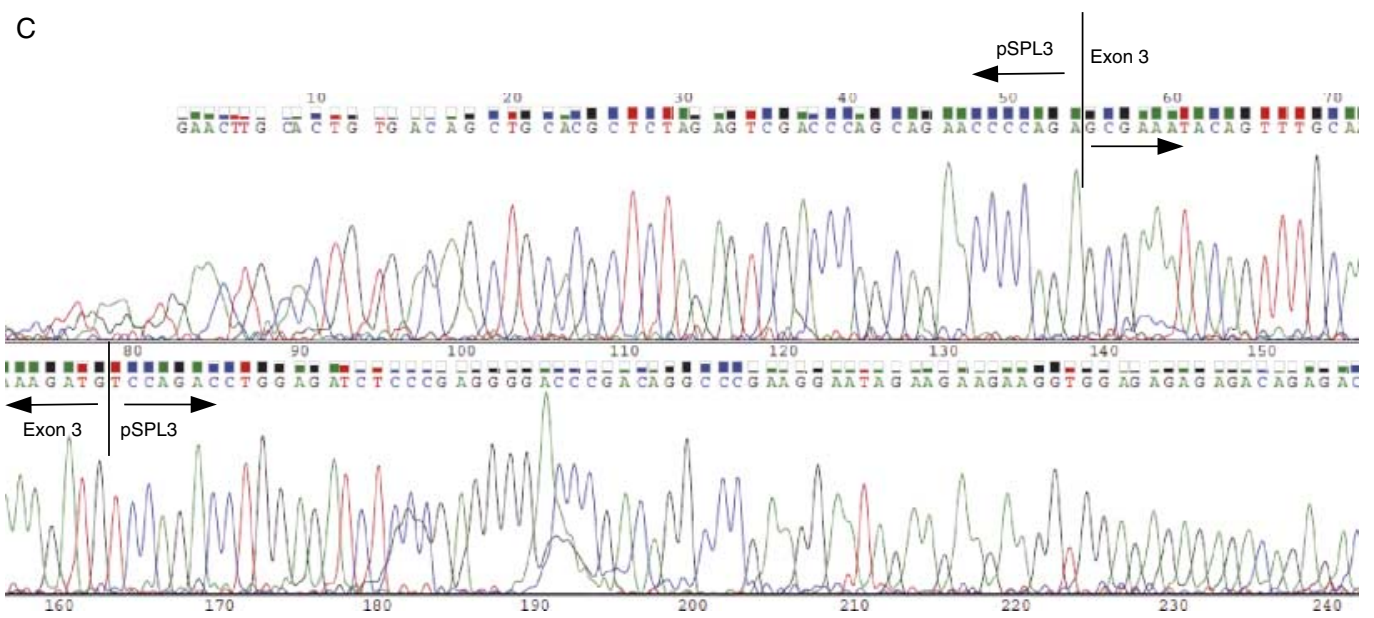

Figure 2 Pre-mRNA splicing of TNFRSF11B as affected by SNP rs4876869. (A) Schematic diagram of construct in pSPL3 exon-trapping vector showing position of rs4876869; SD, splice donor; SA, splice acceptor; (B) agarose gel electrophoresis from all three cell lines used; (C) electropherogram showing DNA sequence of $295 \mathrm{bp}$ transcript. Full colour version of this figure available via http://dx.doi.org/10.1530/JME-11-0067.

statistically significant differences in BMD between genotypes with a statistical power of at least $80 \%$.

In vitro analysis of the $\mathrm{T} / \mathrm{C}$ change in exon 2 (rs4876869) showed that the $\mathrm{C}$ allele affects premRNA processing resulting in transcription of two transcripts, one of which lacks exon 3. Exon 3 encodes for a portion of domain III and entire domain IV, each having a number of cysteine residues forming two or three disulphide bridges. Mutations within these domains can affect disulphide bond formation, thus disrupting OPG protein folding, destabilising its structure and possibly affecting its affinity to RANKL (Cundy et al. 2002). In this study, haplotype analysis shows that SNP C950T is the main contributor to the risk for low BMD in this group of postmenopausal women, since haplotypes harbouring the C950Tallele C are protective, irrespective of which allele for SNP rs4876869 is present. Moreover, haplotype T-G-T increases the risk for lower BMD even though the $\mathrm{T}$ allele for SNP rs4876869 yields only a full-length OPG transcript.

Genetic variations within the TNFRSF11B, RANK and $R A N K L$ genes have been widely studied not only for association with osteoporosis (Liu et al. 2010, Roshandel et al. 2010) but also with other disorders such as rheumatoid arthritis (Tan et al. 2010), Paget's disease of bone (Whyte 2006), cardiovascular disease and cancer metastasis (Hanada et al. 2011, Santini et al. 2011). The RANK/RANKL/OPG system plays a very important role in the immune system cross-linking it to bone in what is known as osteoimmunology and evident in disorders such as rheumatoid arthritis. The treatment using 
exogenous OPG aimed to block both RANKL- and tumour necrosis factor (TNF)-related apoptosis-inducing ligand (TRAIL) is beneficial not only to treat osteoporosis but also for diseases such as multiple myeloma, vascular disease and metastatic breast and prostate carcinomas (Fili et al. 2009). In their review, Fili et al. (2009) discussed how outcomes of in vitro studies can differ from what is observed in vivo such as in the case of breast cancer. In vitro breast cancer cells secrete OPG as self-protection from TRAIL-induced apoptosis but at the same time preventing bone metastasis when mice were treated with high-dose OPG. However, care should be taken when treating multiple myeloma with OPG since this can protect cancerous myeloma cells from TRAIL-induced apoptosis. RANKL blocked by giving exogenous OPG can be beneficial to treat patients with vascular disease, rheumatoid arthritis, fracture healing and osteoporosis. Treatment of osteoporosis with strontium ranelate improves bone formation and decreases osteoclast bone resorption partly by increasing OPG expression and decreasing RANKL (Fonseca 2008, Atkins et al. 2009).

Our study is the first to report functional effects of SNPs within the TNFRSF11B gene promoter and their influence on BMD by looking at two common SNPs in strong LD to each other. SNP C950T that affects gene expression seems to have a dominant effect over SNP rs4876869 that could result in a less effective OPG isoform. This suggests that C950T alleles can be better markers for an increased risk for low BMD in Maltese postmenopausal women.

\section{Declaration of interest}

The authors declare that there is no conflict of interest that could be perceived as prejudicing the impartiality of the research reported.

\section{Funding}

This project was supported by the Research Fund Committee of the University of Malta.

\section{Author contribution statement}

C V contributed to experimental design, data analyses, literature search and writing of this article. R F performed experiments. A X-A is project co-ordinator and co-author of this article.

\section{Acknowledgements}

We would like to thank Dr Anthony Fenech, PhD, B.Pharm. (Hons.), for the use of facilities at the Department of Pharmacology and Clinical Therapeutics, University of Malta, and Prof. Junko Oshima, Department of Pathology, University of Washington, Washington, USA, who generously donated the pSPL3 plasmid.

\section{References}

Atkins GJ, Welldon KJ, Halbout P \& Findlay DM 2009 Strontium ranelate treatment of human primary osteoblasts promotes an osteocyte-like phenotype while eliciting an osteoprotegerin response. Osteoporosis International 20 653-664. (doi:10.1007/ s00198-008-0728-6)

Boyle WJ, Simonet WS \& Lacey DL 2003 Osteoclast differentiation and activation. Nature 423 337-342. (doi:10.1038/nature01658)

Brandstrom H, Gerdhem P, Stiger F, Obrant KJ, Melhus H, Ljunggren O, Kindmark A \& Akesson K 2004 Single nucleotide polymorphisms in the human gene for osteoprotegerin are not related to bone mineral density or fracture in elderly people. Calcified Tissue International 74 18-24. (doi:10.1007/s00223-002-2136-9)

Cundy T, Hegde M, Naot D, Chong B, King A, Wallace R, Mulley J, Love DR, Seidel J, Fawkner M et al. 2002 A mutation in the gene TNFRSF11B encoding osteoprotegerin causes an idiopathic hyperphosphatasia phenotype. Human Molecular Genetics 11 2119-2127. (doi:10.1093/hmg/11.18.2119)

Fili S, Karalaki M \& Schaller B 2009 Therapeutic implications of osteoprotegerin. Cancer Cell International 9 26. (doi:10.1186/14752867-9-26)

Fonseca JE 2008 Rebalancing bone turnover in favour of formation with strontium ranelate: implications for bone strength.

Rheumatology 47 (Supplement 4) iv17-iv19. (doi:10.1093/rheumatology/ken165)

García-Unzueta MT, Riancho JA, Zarrabeitia MT, Sañudo C, Berja A, Valero C, Pesquera C, Paule B, González-Macías J \& Amado JA 2008 Association of the $163 \mathrm{~A} / \mathrm{G}$ and $1181 \mathrm{G} / \mathrm{C}$ osteoprotegerin polymorphism with bone mineral density. Hormone and Metabolic Research 40 219-224. (doi:10.1055/s-2008-1046793)

Hagedorn C, Telgmann R, Dördelmann C, Schmitz B, Hasenkamp S, Cambien F, Paul M, Brand E \& Brand-Herrmann SM 2009 Identification and functional analyses of molecular haplotypes of the human osteoprotegerin gene promoter. Arteriosclerosis, Thrombosis, and Vascular Biology 10 1638-1643. (doi:10.1161/ATVBAHA. 109.193532)

Hanada R, Hanada T, Sigl V, Schramek D \& Penninger JM 2011 RANKL/RANK-beyond bones. Journal of Molecular Medicine 89 647-656. (doi:10.1007/s00109-011-0749-z)

Hofbauer LC \& Schoppet M 2002 Osteoprotegerin gene polymorphism and the risk of osteoporosis and vascular disease. Journal of Clinical Endocrinology and Metabolism 87 4078-4079. (doi:10.1210/jc.2002-021063)

Hofbauer LC, Khosla S, Dunstan CR, Lacey DL, Boyle WJ \& Riggs BL 2000 The roles of osteoprotegerin and osteoprotegerin ligand in the paracrine regulation of bone resorption. Journal of Bone and Mineral Research 15 2-12. (doi:10.1359/jbmr.2000.15.1.2)

Jurado S, Nogués X, Agueda L, Garcia-Giralt N, Urreizti R, Yoskovitz G, Pérez-Edo L, Saló G, Carreras R, Mellibovsky L et al. 2010 Polymorphisms and haplotypes across the osteoprotegerin gene associated with bone mineral density and osteoporotic fractures. Osteoporosis International 21 287-296. (doi:10.1007/s00198-0090956-4)

Liu JM, Zhang MJ, Zhao L, Cui B, Li ZB, Zhao HY, Sun LH, Tao B, Li M \& Ning G 2010 Analysis of recently identified osteoporosis susceptibility genes in Han Chinese women. Journal of Clinical Endocrinology and Metabolism 95 E112-E120. (doi:10.1210/jc.20092768)

Mencej S, Albagha OME, Prezelj J, Kocjan T \& Marc J 2008 Tumour necrosis factor superfamily member 11 gene promoter polymorphisms modulate promoter activity and influence bone mineral density in postmenopausal women with osteoporosis. Journal of Molecular Endocrinology 40 273-279. (doi:10.1677/JME-08-0003)

Park OJ, Shin SY, Choi Y, Kim MH, Chung CP, Ku Y \& Kim KK 2008 The association of osteoprotegerin gene polymorphisms with periodontitis. Oral Disease 14 440-444. (doi:10.1111/j.1601-0825.2007. 01398.x) 
Richards JB, Rivadeneira F, Inouye M, Pastinen TM, Soranzo N, Wilson SG, Andrew T, Falchi M, Gwilliam R, Ahmadi KR et al. 2008 Bone mineral density, osteoporosis, and osteoporotic fractures: a genome-wide association study. Lancet 371 1505-1512. (doi:10. 1016/S0140-6736(08)60599-1)

Roshandel D, Holliday KL, Pye SR, Boonen S, Borghs H,

Vanderschueren D, Huhtaniemi IT, Adams JE, Ward KA, Bartfai G et al. 2010 Genetic variation in the RANKL/RANK/OPG signaling pathway is associated with bone turnover and bone mineral density in men. Journal of Bone and Mineral Research 25 1830-1838. (doi:10. 1002/jbmr.78)

Santini D, Perrone G, Roato I, Godio L, Pantano F, Grasso D, Russo A, Vincenzi B, Fratto ME, Sabbatini R et al. 2011 Expression pattern of receptor activator of $\mathrm{NF \kappa B}$ (RANK) in a series of primary solid tumors and related bone metastases. Journal of Cellular Physiology 226 780-784. (doi:10.1002/jcp.22402)

Styrkarsdottir U, Halldorsson BV, Gretarsdottir S, Gudbjartsson DF, Walters GB, Ingvarsson T, Jonsdottir T, Saemundsdottir J, Center JR, Nguyen TV et al. 2008 Multiple genetic loci for bone mineral density and fractures. New England Journal of Medicine 358 2355-2365. (doi:10.1056/NEJMoa0801197)

Tan W, Wu H, Zhao J, Derber LA, Lee DM, Shadick NA, Conn DL, Smith EA, Gersuk VH, Nepom GT et al. 2010 A functional RANKL polymorphism associated with younger age at onset of rheumatoid arthritis. Arthritis and Rheumatism 62 2864-2875. (doi:10.1002/art. 27589)
Terwillinger JD \& Ott J 1994 Linkage disequilibrium between alleles at marker loci. In Handbook of Human Genetic Linkage, pp 188-198. Baltimore: John Hopkins University Press.

Ueland T, Bollerslev J, Wilson SG, Dick IM, Islam FM, Mullin BH, Devine A \& Prince RL 2007 No associations between $O P G$ gene polymorphisms or serum levels and measures of osteoporosis in elderly Australian women. Bone 40 175-181. (doi:10.1016/j.bone. 2006.06.022)

Vidal C, Brincat M \& Xuereb Anastasi A 2006 TNFRSF11B gene variants and bone mineral density in postmenopausal women in Malta. Maturitas 53 386-395. (doi:10.1016/j.maturitas.2005.11.003)

Vidal C, Cachia A \& Xuereb-Anastasi A 2009 Effects of a synonymous variant in exon 9 of the $C D 44$ gene on pre-mRNA splicing in a family with osteoporosis. Bone 45 736-742. (doi:10.1016/j.bone.2009.06. 027)

Whyte MP 2006 Paget's disease of bone and genetic disorders of RANKL/OPG/RANK/NF-kappaB. Annals of the New York Academy of Sciences 1068 143-164. (doi:10.1196/annals.1346.016)

Xu XH, Dong SS, Guo Y, Yang TL, Lei SF, Papasian CJ, Zhao M \& Deng HW 2009 Molecular genetic studies of gene identification for osteoporosis: the 2009 update. Endocrine Reviews 31 447-505. (doi:10.1210/er.2009-0032)

Received in final form 26 August 2011

Accepted 12 October 2011

Made available online as an Accepted Preprint 12 October 2011 